Revista ELectrónica de Investigación y EValuación Educativa

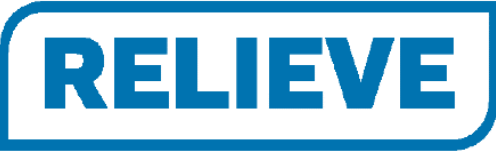

e-Journal of Educational Research, Assessment and Evaluation

ISSN: 1134-4032

\title{
Las prácticas externas desde la perspectiva de las entidades colaboradoras
} External placements from the perspective of collaborating companies

\author{
Ferrández-Berrueco, Reina \& Sánchez-Tarazaga, Lucía \\ Universitat Jaume I (España)
}

\begin{abstract}
Introduction: The partnership between higher education and the labour market is a prerequisite for improving graduates' employment opportunities. However, this partnership is not always as fluid as it should be, sometimes because universities do not understand what companies expect from the collaboration and forget that companies are not NGOs; other times because companies are not aware of the potential benefits and impacts they can derive from the partnership. This paper sheds light on the perception of this collaboration held by the entities collaborating with the university in placements, and uncovers their motivations and demands. Methodology: Based on the literature review of motivations, impacts and benefits associated with university-business partnerships, semi-structured interviews were conducted with a sample of entities collaborating in external practices with Jaume I University, following a cuota sampling design. Results: The main results obtained from the content analysis show that the main motivations driving entities to collaborate are the principles of corporate social responsibility, although underlying this motivation is the selection of potential future employees. The demands organisations made include improving the quality, quantity and forms of relationship between the partners, and the opportunity to enjoy the benefits of using university services. Discussion: In the field of Spanish academia, the collaboration between university and business generally seems to be discussed as a oneway relationship. Universities show a certain lack of interest in partnerships, and their concern appears to focus more on assigning students to placements rather than on their specific suitability. In addition, work placement practices are often designed by academics who fail to consider the real needs of the labour market.
\end{abstract}

Keywords: External placements, higher education, education work relationship, corporate social responsibility.

\section{Resumen}

Introducción: La cooperación entre la educación superior y el mercado laboral es un requisito para mejorar las oportunidades de empleo de los titulados. Sin embargo, esta asociación no siempre es tan fluida como debería, a veces porque las Universidades no entienden lo que las entidades esperan de esa colaboración y olvidan que éstas también deben ser compensadas de alguna manera. Pero otras veces las empresas no son conscientes de los beneficios potenciales y el impacto que pueden obtener de esta colaboración. El presente trabajo hace un acercamiento a la percepción que las entidades que colaboran con la universidad en prácticas externas tienen de dicha colaboración, así como sus motivaciones y demandas. Metodología: A partir de la revisión de la literatura al respecto de motivaciones, impactos y beneficios asociados a la colaboración entre universidad y empresa, se ha realizado una entrevista semiestructurada a una muestra de entidades colaboradoras en prácticas externas de la Universidad Jaume I siguiendo un muestreo de cuotas. Resultados: A partir del análisis de contenido, los resultados muestran que las entidades colaboran sobre todo movidas por principios de Responsabilidad Social Corporativa, aunque subyacente a esa motivación aparece la selección de potenciales futuros empleados como base de la colaboración. En el terreno de las demandas, aparecen el de mejorar la calidad, cantidad y formas de relación entre ambas organizaciones, así como la posibilidad de disfrutar de beneficios en la utilización de servicios universitarios. Discusión: Al hablar de colaboración entre universidad y empresa para la docencia en nuestro país, en términos generales, parece hablarse de relación unidireccional. Las universidades muestran un cierto desinterés por mantener y fidelizar dicha

\section{Reception Date}

2018 October 10

Approval Date

2019 May 04

Publication Date: 2019 May 04 
relación. De hecho, esta preocupación parece estar más vinculada a la localización de los puestos de prácticas que a la idoneidad específica de estos. Además, dichas prácticas suelen estar diseñadas únicamente por profesorado universitario no teniendo en cuenta las necesidades reales del mercado laboral.

Palabras clave: Prácticas externas, educación superior, relación educación empleo, responsabilidad social corporativa

La estrategia Europa 2020 pone a los sistemas educativos en el punto de mira para conseguir un crecimiento más sostenible e inclusivo (Comisión Europea, 2010). Todo esto obliga a desarrollar una formación más relevante para la sociedad, nuevas formas de impartir dicha formación, así como nuevos modos de validar y evaluar el aprendizaje.

En el centro de este objetivo yace la necesidad imperiosa de establecer puntos de unión entre las universidades y las entidades externas $^{1}$ como medio de mejorar la relevancia de la formación y facilitar el acceso al mundo laboral (Comisión Europea, 2011a). Más concretamente, tal y como pone de manifiesto un informe de la OCDE (2012), la estrecha colaboración de estas a través de diferentes proyectos permite oportunidades de adquirir competencias a sus estudiantes. Así, la conexión entre instituciones ya ha sido ampliamente defendida, especialmente en lo que se refiere a las prácticas externas. Pero no solo por su carga lectiva en el plan de estudios, sino por erigirse como un periodo de transición clave entre la formación académica y el entorno laboral, por cuanto supone una socialización profesional del estudiante y su habilitación para el ejercicio de la profesión. De este modo, el alumnado puede ejercitarse durante el mismo en un contexto similar al que se encontrará en el futuro, apropiándose de marcos de referencia para desarrollar y adquirir competencias profesionales (Álvarez, Iglesias \& García, 2007; Egido, 2017; Latorre \& Blanco, 2011; Valle \& Manso, 2011).

Además, los procesos de aprendizaje arraigados al entorno laboral que ofrecen las instituciones y empresas ayudan a la universidad a interpretar y responder pedagógicamente a los retos de la sociedad, cada vez más inciertos y complejos.

Sin embargo, el análisis de la situación europea en este momento, y en especial, la de nuestro país, sugiere que los sistemas educativos (especialmente los universitarios) no están respondiendo con la celeridad necesaria a estos cambios, fracasando en la adaptación de los programas a las necesidades de la economía y el mercado laboral.

En ocasiones, las universidades no entienden lo que las empresas esperan de esa colaboración y olvidan que las empresas buscan algún tipo de compensación. Pero otras veces las empresas no son conscientes de los beneficios potenciales y el impacto que pueden obtener de esta colaboración (Ferrández et al. 2016a; 2016b).

En cualquier caso, la colaboración es necesaria y, para que esto ocurra de una forma significativa y sostenible, es fundamental que las universidades entiendan lo mejor posible cómo animar y maximizar el potencial de este tipo de cooperación. Esto requiere, de manera explícita que la universidad entienda las motivaciones, beneficios e impacto potencial percibido por dichas organizaciones.

Para ello es necesario responder a los siguientes interrogantes: ¿Qué motiva a una empresa colaborar con la universidad? ¿Qué beneficios espera obtener? ¿Cuál es o ha sido el impacto esperado y recibido de dicha colaboración?

La revisión de la literatura nos muestra posibles respuestas a estos interrogantes desde el ámbito internacional. Estas respuestas las hemos clasificado en cuatro

\footnotetext{
${ }^{1}$ A lo largo del trabajo, para no resultar repetitivo, se utilizarán indistintamente los términos, entidad, empresa, empleador u organización, pero siempre han de entenderse en su sentido más amplio de representantes del mundo laboral.
} 
tipos diferentes de motivaciones, impactos o beneficios: de tipo económico, de responsabilidad social corporativa (RSC), de innovación y de tipo estratégico.

Variables económicas. Estos factores están relacionados con la percepción del incremento de la productividad de la entidad (Basit et al. 2015; Daley et al. 2016) y el ahorro, tanto en términos de formación de los empleados (García Delgado, 2002; Guinart, 2005; Healy et al., 2014; Marzo et al. 2008) como obtener recursos humanos gratuitos o a muy bajo coste por algún tiempo (Elijido-Ten \& Kloot, 2015; Ferrández-Berrueco, et al., 2016a; Siebert \& Costley, 2013).

Otras veces, cuando la colaboración no se reduce a las prácticas externas, el ahorro se refiere a compartir y reducir gastos en $\mathrm{I}+\mathrm{D}$ (Marzo et al. 2008).

Responsabilidad Social Corporativa (RSC). La RSC se refiere a las empresas que asumen responsabilidad por su impacto en la sociedad (Comisión Europea, 2011b), un concepto transversal que afecta a diferentes ámbitos de la gestión de las empresas. Estos factores están relacionados con la percepción de la imagen social de la empresa, la reputación y la mejora del prestigio al ayudar a la universidad (Daley et al. 2016, FerrándezBerrueco et al., 2016 ${ }^{\text {a }}$ 2016b; Healy et al., 2014; Marzo et al., 2008).

Esta mejora de imagen proporciona buena propaganda a la empresa (FerrándezBerrueco et al., 2016b), pero también ofrece la oportunidad de influir en el currículo de las universidades, lo que proporciona mejores programas a los estudiantes para mejorar su empleabilidad y mejorar las prestaciones sociales. (Ferrández-Berrueco et al., 2016b; Garnett, 2016; Marzo et al., 2008; Whittington \& Ferrández, 2007).

Transformación. Bajo este concepto se abordan diferentes enfoques, siendo el punto en común de todos ellos que la colaboración permite a la empresa actualizarse y promueve una actitud emprendedora (Healy et al., 2014).

De esta forma encontramos argumentos que hablan de la modernización de los empleados
(Basit et al. 2015; Felce, 2017; FerrándezBerrueco, 2016a; Marzo et al. 2008; Whittington \& Ferrández, 2007) y del aprendizaje de nuevos conocimientos y las últimas innovaciones que los empleados pueden adquirir a través de estudiantes en prácticas (Antcliff et al. 2016; Geller et al. 2016; Guinart, 2005). Esto último implica que además se promueva la incorporación de la cultura del aprendizaje (White, 2012) introduciendo el ciclo de mejora continua (Ions \& Minton 2012).

Por otra parte, encontramos otros factores relacionados con la reflexión profesional en cuanto que la colaboración permite una mejor aceptación de los cambios y obliga al entendimiento mutuo (White, 2012), haciendo que la empresa se aleje de lo tradicional (Whittington \& Ferrández, 2007; Ions \& Minton 2012). En resumen, la colaboración transforma a las empresas en organizaciones de aprendizaje (Bolívar, 2007; Ions \& Minton, 2012), un concepto que ya introdujo Peter Senge (1992) en el ámbito empresarial y que con posterioridad se ha incorporado al contexto educativo

Finalmente, dentro de este apartado hemos incluido los factores relacionados con la evaluación, puesto que la colaboración proporciona a la empresa un referente para evaluar su eficiencia (Hegarty et al. 2011) y al mismo tiempo es una forma de evaluar el desempeño de los empleados (Siebert \& Costley, 2013) promoviendo así una mejor cualificación de los empleados actuales y futuros (Felce et al. 2016).

Planificación estratégica. Este último grupo de beneficios se relaciona principalmente con dos perspectivas. Por un lado, la planificación estratégica entendida como prospectiva. Así, colaborar con la universidad y, más concretamente, acoger estudiantes en prácticas, facilita la identificación de nuevos perfiles profesionales (Guinart, 2005) y la selección de nuevos empleados (Daley et al. 2016; Elijido-Ten \& Kloot, 2015; Felce 2017; Ferrández-Berrueco et al. 2016a; García Delgado 2002; Healy et al., 2014; Marzo et al. 2008; Whittington \& Ferrández, 2007), así como su fidelidad (Daley et al. 2016). 
Por otra parte, la planificación estratégica también hace referencia a la posibilidad de obtener buenos contactos en la universidad (Geller et al. 2016; Healy et al. 2014; Hegarty et al. 2011) ya que la universidad es una fuente de conocimiento y tecnología para las empresas (Marzo et al. 2008).

\section{Objetivos}

Una vez respondidas desde la literatura las motivaciones y beneficios que las entidades pueden obtener y esperar cuando colaboran con la universidad, queda una segunda parte: verificar en la práctica las respuestas reales que proporcionan las entidades colaboradoras de nuestro entorno. Así, el objetivo principal que planteamos en este estudio ${ }^{22}$ es averiguar cuáles de las motivaciones y beneficios que se asocian a la cooperación son las que están en la mente de las entidades que ya colaboran en las prácticas. En segundo lugar, de manera asociada a este objetivo, pretendemos averiguar qué elementos o variables se perciben como barreras para una mayor colaboración. Por último, como cuestión inseparable del objetivo anterior, intentamos encontrar qué factores externos a las motivaciones y beneficios iniciales detectados en el primer objetivo podrían facilitar o promover una mejor relación.

\section{Método}

Con el fin de responder a este objetivo, y teniendo en cuenta el carácter exploratorio de este trabajo debido a la poca literatura existente en nuestro idioma (véase, por ejemplo, Bolívar, 2007; García Delgado, 2002; Guinart, 2005; Marzo, Pedraja \& Rivera, 2008; Zabalza, 2011, 2013) se optó por realizar una entrevista semiestructurada (ver Tabla 1) a un conjunto de entidades que representaran las diferentes tipologías que ya participaban en las prácticas externas acogiendo estudiantes de las diferentes titulaciones impartidas. Se optó por este tipo de entrevista para intentar combinar una doble finalidad: confirmar la teoría recogida desde la literatura internacional y validarla dentro de nuestro contexto (Wengraf, 2012).

El borrador de la entrevista fue realizado por el equipo de investigación a partir de la revisión bibliográfica y las categorías resultantes y fue validado por tres expertos internacionales en el ámbito de las relaciones universidad-empresa ${ }^{3}$. Tras su revisión, nos recomendaron incluir una cuestión relativa a la vinculación previa con la universidad que tenía la persona de la entidad que había decidido acoger estudiantes de prácticas. Esta variable resultaba relevante a la hora de analizar la motivación de colaboración.

La recogida de datos fue realizada en noviembre de 2017 por parte de seis entrevistadores, miembros del equipo de trabajo, a los que se les formó durante dos meses sobre la cuestión. Estos contactaron, vía telefónica, con el responsable de cogida del alumnado de la universidad, que fue quien respondió a la entrevista. Las respuestas se registraron en un documento Word para, posteriormente, proceder con el análisis de contenido. Este análisis se realizó siguiendo las propuestas de varios autores (ÁlvarezGayou, 2005; Fernández, 2006; Miles \& Huberman, 1994) y permitió reducir la información en categorías de contenido, cuyo proceso interpretativo se aborda en el apartado de resultados del presente artículo.

\footnotetext{
${ }^{2}$ Los resultados de esta investigación forman parte del proyecto Relaciones Universidad-Empresa para docencia. Motivaciones, Impacto y Beneficio. EMBI (UJI-B2016-04).

${ }^{3}$ Los expertos fueron: Tauno Kekäle, rector de la Universidad de Ciencias Aplicadas de Vasa (Finlandia),
}

Stefan Humpl (Austria), experto designado por la Unión Europea para el fomento de las relaciones universidadEmpresa y Jayne Mothersdale, responsable del desarrollo de contratos de aprendizaje en las empresas para la obtención de títulos universitarios en la Leeds Beckett University (Reino Unido). 
Tabla 1. Entrevista semiestructurada. Información general

\section{A. DATOS PERSONALES}

Relación previa personal con la universidad

Cargo del entrevistado

Tiempo que lleva colaborando con la universidad

\section{B. PREGUNTAS GENERALES}

1. ¿Por qué empezaron a trabajar con Universidad?

2. ¿De quién surgió la iniciativa?

3. ¿Qué relación previa tenía con la Universidad?

4. ¿Con cuántos alumnos/as suelen contar?

5. ¿Cuáles son las razones por las que sigue colaborando?

6. ¿La información brindada desde la universidad, con respecto a las posibilidades de la colaboración hacia su empresa, es suficiente? ¿Por qué? ¿Qué echa de menos?

7. ¿Le gustaría beneficiarse de algún servicio de la universidad por el hecho de estar colaborando con ella?

8. ¿QQué le aporta el alumnado de prácticas a la empresa?

9. ¿Mantienen algún otro contacto, además de las prácticas, con la universidad? Por ejemplo, dar charlas...

10. En algunos países europeos, las entidades colaboran más allá de las prácticas, por ejemplo: Colaboran en el diseño de planes de estudio, proporcionan casos reales para que los estudiantes los trabajen, solicitan cursos a la universidad para reciclar o actualizar a los trabajadores. ¿¿Su entidad estaría interesada en este tipo de colaboración? ¿Cuál de los ejemplos que le he dado le parece más atractivo?

11. ¿Cuáles cree que son los principales inconvenientes para que haya una colaboración más estrecha con la universidad?

12. ¿¿ué echa de menos al colaborar con la universidad?

13. ¿Por qué acoge alumnos de distintos perfiles? (solo perfil variado)

14. ¿Tiene alguna motivación especial? (solo perfil variado)

15. ¿Quién lo decide? (solo perfil variado)

\section{PREGUNTAS SOBRE FACTORES ECONÓMICOS}

1. Al tener alumnado de prácticas, ¿Cree que los gastos en la formación de los empleados/as disminuyen?

2. ¿ ¿Piensa que colaborando con la universidad podría aumentar la productividad de la empresa? ¿Por qué?

3. ¿Cuál es el mayor riesgo económico que cree que puede ocurrir al colaborar con la universidad?

\section{PREGUNTAS SOBRE RSC}

1. ¿Cree que mejora la imagen al colaborar con la universidad? ¿Por qué?

2. ¿¿Recomendaría la colaboración con la universidad a otras entidades? ¿Por qué?

\section{E. PREGUNTAS SOBRE INNOVACIÓN}

1. ¿En qué medida cree que colaborar con la universidad ayuda a la modernización de la empresa?

2. ¿Considera que los estudiantes aportan ideas innovadoras? ¿Por qué?

3. ¿Es usted supervisor de prácticas en la empresa?

4. (Sólo en el caso de que el contacto sea supervisor) ¿Ser supervisor de estudiantes en prácticas le ayuda a aprender en su puesto de trabajo?

\section{F. PREGUNTAS SOBRE PLANIFICACIÓN ESTRATÉGICA}

1. ¿ ¿Utiliza las prácticas externas como mecanismo de selección de personal? ¿Por qué?

2. ¿Han contratado a estudiantes de la universidad que hayan hecho las prácticas en su empresa? ¿Cómo se llegó a este contrato? ¿Se le hizo una entrevista o se le llamó directamente?

3. ¿ ¿Se han planteado acoger estudiantes de otro perfil? ¿Por qué?

\section{CONCLUSIÓN}

¿Desea añadir algo más?

\section{Muestra}

Para la selección de las entidades a entrevistar, se solicitó a la universidad la base de datos de empresas colaboradoras en prácticas externas durante el curso 2016/2017 que resultaron un total de 922 (ver Tabla 2). Se procedió a su actualización y clasificación atendiendo a las categorías que se consideraron relevantes para el estudio y que permitirían realizar una estratificación adecuada a la hora de realizar el muestreo para las entrevistas. Son las siguientes: 
Tamaño: Se dividieron en cuatro categorías, atendiendo a la definición de la UE (DOUE, 2003): Grande ( $\geq 250$ empleados), Mediana (249-50), Pequeña (49-10) y Microempresas ( $<10$ empleados).

Sector de producción: Para el sector de producción se ha utilizado la definición más extendida en cuatro sectores (Kenessey, 1987): Primario (agricultura, pesca y ganadería), Secundario (industria, construcción y manufactura), Terciario (comercio y servicios), Cuaternario (gestión de la información y la tecnología).

Sector académico: Según su ámbito de producción, se determinó, en la medida de lo posible, el área académica dominante según establece el RD1393/2007 (Artes y Humanidades, Ciencias Sociales y Jurídicas, Ciencias, Salud, Ingeniería y Arquitectura, Otros).

Perfil de estudiantes: Se estableció una categorización de tipo dicotómico, en la que se señalaba "perfil único" cuando solo acogían estudiantes de una titulación y "perfil variado" cuando acogían estudiantes de varias titulaciones.

Titularidad: Se establecieron dos tipos de titularidades, atendiendo a la propiedad del capital: Pública y Privada.

Una vez establecido el perfil de empresas, se procedió a realizar la selección de la muestra para las entrevistas. Se optó por un muestreo no probabilístico mixto de elección experta y por cuotas (Kalton, 1983) de tal manera que nos asegurase al menos una empresa de cada una de las modalidades anidadas, seleccionando algunos casos específicos cuando la información que podían proporcionar era más amplia por colaborar con la universidad de manera cotidiana en otros asuntos. Así, por ejemplo, en los casos en los que el subconjunto de entidades de un perfil determinado incluía algún patrocinador o entidad con un peso específico en la universidad, esta era automáticamente seleccionada para la entrevista. Del mismo modo, se tuvo en cuenta también seleccionar algún representante de casos muy especiales que podían ser enmascarados dentro de un subgrupo más homogéneo. Era el caso de ONGs o Asociaciones por ejemplo con muy escasa representación en la población de referencia y que podían quedar ocultas dentro de las entidades privadas pequeñas o micro del sector terciario.

De este modo, el total de entidades a entrevistar quedó fijado en 72. Esta cantidad se redujo, finalmente, a 46 (ver Tabla 2) puesto que la información recogida dejaba aportar nuevos elementos, alcanzándose la saturación (Hernández Carrera, 2014)

Tabla 2. Resumen de entidades colaboradoras atendiendo a las variables consideradas relevantes para el muestreo $(\mathrm{N})$ y composición de la muestra $(\mathrm{n})$

\begin{tabular}{clcc}
\hline VARIABLE & CATEGORÍA & N (\%) & n (\%) \\
\hline \multirow{4}{*}{ Tamaño } & Grande (G) & $96(10,7)$ & $11(23,9)$ \\
& Mediana (M) & $144(16,1)$ & $13(28,3)$ \\
& Pequeña (P) & $241(26,9)$ & $10(21,7)$ \\
& Micro (Mi) & $414(46,3)$ & $12(26.1)$ \\
\hline \multirow{5}{*}{ Sector de Producción } & Primario (P) & $4(0,4)$ & $1(2,2)$ \\
& Secundario (S) & $103(11,2)$ & $13(28,3)$ \\
& Terciario (T) & $748(81,3)$ & $25(54,3)$ \\
& Cuaternario (C) & $67(7,3)$ & $7(15,2)$ \\
\hline \multirow{5}{*}{ Sector Académico } & Artes y Humanidades (A) & $144(15,6)$ & $5(10,9)$ \\
& Ciencias Sociales y Jurídicas (J) & $408(44,3)$ & $12(26,1)$ \\
& Ciencias (C) & $195(21,2)$ & $12(26,1)$ \\
& Salud (S) & $73(7,9)$ & $5(10,9)$ \\
& Ingeniería y Arquitectura (I) & $83(9,0)$ & $8(17,4)$ \\
& Otros (O) & $19(2,1)$ & $4(8.7)$ \\
\hline \multirow{2}{*}{ Perfil de Estudiantes } & Único (U) & $872(94,6)$ & $37(80,4)$ \\
& Variado (V) & $50(5,4)$ & $9(19,6)$ \\
\hline \multirow{2}{*}{ Titularidad } & Pública (P) & $129(14,0)$ & $9(19,6)$ \\
& Privada (Pr) & $793(86)$ & $37(80,4)$ \\
\hline
\end{tabular}




\section{Resultados}

Realizado el análisis de contenido de las entrevistas, y una vez constatada la inexistencia de diferencias significativas en los patrones de respuesta según las variables independientes consideradas (tamaño, sector de producción, sector académico, perfil de estudiantes y titularidad), presentamos los principales resultados obtenidos, siguiendo los objetivos planteados en el estudio.

\section{Motivaciones y beneficios}

En primer lugar, pretendíamos averiguar las motivaciones y beneficios que las entidades que ya colaboran con la universidad asocian a la cooperación (tabla 3). Debe tenerse en cuenta que, a la hora de interpretar los resultados, aunque el total de entidades entrevistadas fueron 46, en bastantes ocasiones las respuestas otorgadas respondían a una o varias categorías por lo que el total de respuestas no siempre será 46 sino superior. El porcentaje de cada categoría, no obstante, está calculado sobre las 46 entidades.

Los resultados expuestos van acompañados de comentarios concretos realizados por las entidades entrevistadas. Para identificar el perfil de la entidad hemos utilizado siguiendo el mismo orden de la tabla 2, las iniciales de cada una de las categorías consideradas y que se han puesto entre paréntesis. De este modo, cuando al lado de un comentario aparezca, por ejemplo (GTJUP), significará que ese comentario ha sido realizado una empresa Grande, de sector Terciario, del ámbito Jurídico y Social que solo acoge estudiantes de un único perfil y es de titularidad Pública.

Tabla 3. Principales resultados del análisis de contenido de las entrevistas

\begin{tabular}{clcc}
\hline VARIABLE & CATEGORÍA & $\mathbf{n}$ & \% \\
\hline \multirow{3}{*}{ Motivación para colaborar } & Planificación estratégica & 6 & 13,6 \\
& I+D & 1 & 2,3 \\
& RSC & 37 & 84.1 \\
& Ns/nc & 2 & 4,3 \\
\hline & Ahorro en salarios & 7 & 15,2 \\
Beneficios de tipo Económico & Es una inversión de futuro & 14 & 30,4 \\
& Aumenta la productividad & 25 & 54,3 \\
& Ninguno & 8 & 17,4 \\
\hline Beneficios asociados a la RSC & Mejora de la imagen & 44 & 95,7 \\
& Propaganda de la entidad & 11 & 23,9 \\
Beneficios asociados a la & Ninguno & 7 & 15,2 \\
\hline Transformación de la empresa & Innovación/modernización & 31 & 67,4 \\
& Aprendizaje & 14 & 30,4 \\
& Cambio & 6 & 13,0 \\
Blanificación estratégica & Ninguno & 8 & 17,4 \\
\hline & Influir en el curriculum & 3 & 6,5
\end{tabular}

A la vista de los resultados, el primer dato que llama la atención es que la gran mayoría de las entidades $(84,1 \%)$ alega motivaciones relacionadas con la RSC para colaborar con la universidad. Argumentos del tipo: "es bueno para la empresa y para los estudiantes" (MiSJUPr) "la universidad nos pidió colaboración” (GTJUP); "los alumnos vinieron a buscarnos" (GSIUPr) etc. Son ejemplos de comentarios que se han englobado en esta categoría. En segundo lugar, la motivación se asocia a la planificación estratégica, siendo la necesidad de sondear la calidad de los futuros graduados la justificación más mencionada (13,6\%). 
Sin embargo, al profundizar en cada una de las variables que mencionaba la literatura, puede apreciarse inmediatamente que si bien la RSC es la motivación que se menciona a modo de escaparate para la colaboración, en el trasfondo aparecen argumentos que señalan claros beneficios para la empresa en todas las modalidades señaladas, esto es Económicas, RSC, Transformación y Planificación estratégica.

Así, dentro de los beneficios de tipo económico se destaca el aumento de la productividad $(54,3 \%)$ porque sale “más trabajo adelante” (MSIUPr) o porque "mientras los estudiantes en prácticas se dedican a las tareas más básicas y mecánicas, los empleados pueden avanzar en tareas más complejas" (GTJUP). Ello, claramente supone utilizar a los estudiantes como mano de obra encubierta lo que se traduce en el ahorro en salarios (15,2\%).

En cuanto a los beneficios asociados a la RSC, la mejora de la imagen $(95,7 \%)$ ha sido la más mencionada, con argumentos del tipo "mejora la posición social de la empresa" (GSAUPr), da "una mayor reputación" (GTJUPr), “demuestra que somos una empresa seria” (MiTOUP). Pero también aparecen destacados otro tipo de beneficios asociados a esta mejora de posición y es el hecho de que la colaboración proporciona propaganda positiva (23,9\%). Por un lado "es una buena publicidad para la empresa” (MSCUPr) y, por otro, "los propios estudiantes hacen propaganda de la entidad” (GSIUPr).

El tercer grupo de beneficios son los asociados a la transformación de la empresa, y en este grupo más de la mitad de las entidades estuvieron de acuerdo en señalar la importancia de la colaboración para modernizar la empresa $(67,4 \%)$ dado que los estudiantes aportan ideas novedosas (MiTJUPr) y, estas ideas novedosas les permiten aprender cosas nuevas (30,4\%).

Por último, el grupo relativo a la planificación estratégica remarca la prospectiva de futuros trabajadores como el beneficio más destacado (69,6\%), lo que encajaría con la motivación oculta bajo la RSC. De hecho, el 65,2\% de las entidades $(\mathrm{n}=30)$ habían contratado a algún alumno de prácticas cuando terminaron los estudios. Teniendo en cuenta que las empresas públicas no tienen capacidad de contratación, y que la muestra contaba con 37 entidades privadas podríamos afirmar que prácticamente todas las entidades con autonomía en la gestión de sus plantillas habían utilizado este sistema como mecanismo de selección de personal $(81,1 \%)$. Algunas, de hecho, realizaron la contratación de manera directa, sin ningún otro mecanismo adicional de selección (GTSUP).

\section{Barreras y facilitadores}

A continuación, presentamos los resultados relacionados con el segundo y tercer objetivo planteados (tabla 4), y que se refieren a las barreras que impiden y factores que facilitarían una mayor colaboración entre la academia y el mundo laboral. Esta información ha sido analizada, principalmente, desde las respuestas proporcionadas a las preguntas 6 a la 12 de la sección general del cuestionario.

Tabla 4. Principales barreras y facilitadores a la colaboración

\begin{tabular}{clcc}
\hline VARIABLE & CATEGORÍA & n & \% \\
\hline \multirow{2}{*}{ Barreras } & Falta de contacto directo & 20 & 43,5 \\
& Falta de información & 18 & 39,1 \\
\hline \multirow{3}{*}{ Facilitadores } & Trato preferente & 17 & 37,0 \\
& Publicitar la empresa & 15 & 32,6 \\
& Proporcionar casos reales & 14 & 30,4 \\
& Diseño de planes de estudio & 7 & 15,2 \\
\hline
\end{tabular}

En lo referente a las principales barreras encontradas, son la falta de contacto directo y la falta de información. En el primer caso, las entidades manifestaban prácticamente (43,5\%) una total falta de contacto con el profesorado de la universidad que realizaba la tutorización 
de los estudiantes de prácticas (MTJUP) lo que deja a las entidades con una cierta sensación de despreocupación por parte de la universidad. En segundo lugar, se demandaba más información (39,5\%), tanto del proceso de prácticas (MTOVPr) como del perfil de los títulos (PSIUPr) o del curriculum del alumnado (MTOVPr). Incluso en varios casos se manifestó desconocimiento en lo referente a los servicios que la propia universidad ofrecía (MTSUP). En el otro extremo ha habido un cierto porcentaje de entidades $(10,9 \%)$ que se han manifestado plenamente satisfechas tanto con el contacto (PTCUPr) como con la información (GTSUPr).

Sin embargo, de las entrevistas también se pudieron extraer otros elementos que hemos calificado como facilitadores, entendiendo que su tratamiento por parte de la universidad permitiría avanzar en la relación. Así, las entidades sugerían, en porcentajes muy similares, obtener algún tipo de trato preferente (37\%), por ejemplo, una reducción de tasas a la hora de acceder a la oferta formativa de la universidad (MiCJUPr) o precios más ventajosos a la hora de alquilar o utilizar las instalaciones (MiSCUPr).

También se mostraban abiertas a entrar en las aulas y colaborar con el profesorado proporcionando casos reales $(30,4 \%)$ que pudieran ser resueltos o debatidos por los estudiantes (GCCUPr) y que, además, servirían para dar a conocer la entidad al estudiantado que después ha de elegir dónde realizar sus prácticas (32,6\%).

Por último, aunque en menor medida, también se mostraban abiertas a otro tipo de colaboraciones como el diseño de los planes de estudio (15,2\%).

\section{Discusión}

Con el fin de establecer puentes entre el curriculum universitario y el entorno laboral y acercarnos así a los objetivos de la Europa 2020 (Comisión Europea, 2011a), el presente trabajo pretendía realizar una primera aproximación a la percepción que las entidades que colaboran con la universidad en prácticas externas tienen de dicha colaboración, así como sus principales motivaciones $\mathrm{y}$ demandas. Solo entendiendo qué es lo que las entidades esperan de dicha cooperación, las universidades podrán establecer mecanismos que mejoren la relación y con ello la calidad de la oferta formativa que ofrecen.

Para ello, se han realizado de una serie de entrevistas semiestructuradas a una muestra de entidades, de todos los perfiles, colaboradoras en las prácticas externas de la universidad Jaume I, pionera en España en establecer como obligatorias las prácticas externas en todos los títulos que ofertaba y con un claro compromiso en su plan estratégico por la transición del alumnado de los estudios al trabajo.

Siguiendo con los objetivos planteados en este estudio, y constatada la inexistencia de diferencias significativas en los patrones de respuesta atendiendo a las variables independientes consideradas, nos detenemos, en primer lugar, en las motivaciones $\mathrm{y}$ beneficios que se asocian a la cooperación de las entidades que ya colaboran en las prácticas. Así, los principales resultados muestran acuerdo con algunas afirmaciones de otras investigaciones de ámbito nacional e internacional, como que las entidades se mueven desde la motivación intrínseca a la hora de colaborar, lo que hemos categorizado bajo la variable $R S C$, buscando una mejora de su posición social (Daley et al. 2016, Ferrández-Berrueco et al., 2016a , 2016b; Healy et al., 2014; Marzo et al., 2008). Pero también colaboran por motivos a medio y corto plazo. Entre los motivos a medio plazo podríamos mencionar los relacionados con la planificación estratégica, más concretamente con la evaluación de posibles futuros empleados (Daley et al. 2016; Elijido-Ten \& Kloot, 2015; Felce 2017; Ferrández-Berrueco et al. 2016a; García Delgado 2002; Healy et al., 2014; Marzo et al. 2008; Whittington \& Ferrández, 2007) y como beneficios más próximos en el tiempo buscarían una mayor productividad, asociado a una variable de tipo económico, aspecto que también ha sido confirmado en otros trabajos (Elijido-Ten \& Kloot, 2015; Ferrández-Berrueco, et al., 2016a; Siebert \& Costley, 2013). 
Alcanzada la saturación, las entrevistas no han mostrado evidencia clara, sin embargo, de otras motivaciones más relacionadas con una relación más estrecha entre ambas organizaciones. Es decir, la empresa busca al estudiante, y solo busca el beneficio o impacto a través de ellos. Así, por ejemplo, ninguna entidad entrevistada ha mencionado que colabora porque la universidad es el centro generador del conocimiento o la pretensión de avanzar hacia convertirse en una organización que aprende (Ions \& Minton, 2012, White, 2012).

En segundo lugar, haremos referencia a las barreras a la colaboración. Así, percibimos que el foco exclusivo en el estudiante es tal, que la gran mayoría de las entidades no eran conscientes de que existían otras formas de colaboración con la universidad, como la posibilidad de entrar en las aulas y contar problemas reales 0 incluso a nivel institucional, la posibilidad de influir en los planes de estudio. Ambos ejemplos, sin embargo, son prácticas muy habituales en otros contextos europeos (Ferrández-Berrueco et al., 2016a, 2016b; Koski, 2017).

Esto, unido a la falta de contacto con el profesorado responsable de las prácticas, manifestada por las entidades, nos lleva a la reflexión de que la universidad no entiende que las prácticas forman parte del proceso formativo y que, como tal, deberían estar coordinadas y planificadas dentro del propio programa, más allá de la mera descripción de duración y requisitos que aparecen en los planes de estudio. Esta denuncia, ya ha sido evidenciada por otros autores en nuestro contexto (Marcelo \& Estebaranz, 1998; Zabalza, 2011, 2013). Pero la realidad parece mostrar que no ha habido un gran cambio a este respecto.

De hecho, el diseño de los programas formativos sigue recayendo, en nuestro país, casi exclusivamente en las universidades, y aunque la legislación vigente señala la necesidad de contactar con los representantes del mundo laboral para su elaboración, la realidad es, que su papel queda reducido a un mero rol administrativo, $\mathrm{y}$ no ven reflejadas realmente sus necesidades (FerrándezBerrueco et al. 2016b).

Además, otro elemento facilitador para la colaboración entre universidad y empresa que ha emergido de este estudio es la utilización de casos reales para la docencia. Esto nos parece particularmente interesante atendiendo que el curriculum universitario está orientado (al menos, en sentido declarativo) hacia las competencias profesionales de sus estudiantes. Esto implica incorporar propuestas metodológicas, diseñadas juntamente con las entidades colaboradoras, donde el aprendizaje del alumnado se encuentre orientado hacia la acción y cuyas actividades tengan una relación directa con el contexto que rodea al estudiante, lo más realistas o auténticas posibles, en palabras de Monereo (2009).

Con la incorporación del EEES en el sistema universitario español se están aplicando con mayor asiduidad metodologías activas como son el aprendizaje basado en proyectos, aprendizaje basado en problemas, aprendizaje servicio o bien seminarios y talleres (De Miguel, 2006). Sin embargo, bajo nuestro punto de vista, no se aprovecha su potencialidad pues se quedan en un nivel de simulación o diseño, olvidando, en muchas ocasiones, la participación de los agentes externos y desaprovechando, en consecuencia, las sinergias fruto de esta colaboración. Sobre modelos o estilos colaborativos pueden encontrarse diversos formatos, lo que parece claro es que no se debe caer en una organización endogámica, sino que hay que fomentar la cooperación, sobre todo para conseguir aprendizajes significativos en los estudiantes (Korthagen, Loughran \& Russell, 2006).

Como adelantábamos en un trabajo anterior (Moliner \& Sánchez-Tarazaga, 2015), no siempre resulta fácil apostar por una colaboración interinstitucional, teniendo en cuenta el modelo organizativo vigente para las propuestas curriculares en la universidad. Éste suele seguir una doble lógica tal y como describe el autor citado anteriormente: por un lado, la de verticalidad (referida a las relaciones de poder) en relación a la toma de 
decisiones y, por otro, a la de fragmentación sucesiva (o especialización) de las propuestas de cada departamento. Con lo que el panorama a la hora de llevar a cabo alguna iniciativa fuera del marco formal se puede convertir en un problema (muchas veces, reducido a lo burocrático), que puede incluso acabar desalentando a los impulsores de la iniciativa.

Por supuesto, esto no significa que las empresas deban dictar a la universidad lo que tiene que hacer, no es su papel (FerrándezBerrueco et al. 2016a), pero tampoco puede dejarse que sea la universidad la que decida todos los elementos del currículo, puesto que en muchas ocasiones los diseños curriculares se mueven más por necesidades estructurales de la propia universidad que por las necesidades del entorno (Major et al., 2011). Por ello es necesario es que se arbitren los mecanismos para que los planes de estudio recojan "seriamente" las necesidades formativas de los futuros graduados, aproximando la formación universitaria a las demandas sociales y del mundo productivo (Zabalza, 2008). Las entidades están abiertas a colaborar, los comentarios que se desprenden de las entrevistas realizadas, así lo indican. Lo único que hace falta es que la universidad les abra la puerta y les deje ver el camino.

\section{Referencias}

Álvarez-Gayou, J.L. (2005). Cómo hacer investigación cualitativa. Fundamentos y metodología. México: Paidós.

Álvarez, E., Iglesias, M. T. \& García, M. S. (2007). Desarrollo de competencias en el Prácticum de Magisterio. Aula Abierta, 36 (1,2), 65-78.

Antcliff, V., Baines, S., \& Gorb, E. (2016). Developing your own graduate employees: Employer perspectives on the value of a degree apprenticeship. Higher Education, Skills and Work-Based Learning, 6(4), 378383. doi: https://doi.org/10.1108/HESWBL05-2016-0032

Basit, T. N., Eardley, A., Borup, R., Shah, H., Slack, K. \& Hughes, A. (2015). Higher education institutions and work-based learning in the UK: employer engagement within a tripartite relationship.
SpringerScience + Business Media

Dordrecht, 70(1), 1003-1015. doi: https://doi.org/10.1007/s10734-015-9877-7

Bolívar, A. (2007). Los centros educativos como organizaciones que aprenden: Promesa y realidades. Madrid: La Muralla.

Comisión Europea (2010). Communication from the Commission Europe 2020. A Strategy for Smart, Sustainable and Inclusive Growth. Recuperado de http://eurlex.europa.eu/LexUriServ/LexUriServ.do?ur i=COM:2010:2020:FIN:EN:PDF

Comisión Europea (2011a). Supporting growth and jobs - an agenda for the modernisation of Europe's higher education systems. Recuperado de http://eurlex.europa.eu/LexUriServ/LexUriServ.do?ur i=COM:2011:0567:FIN:EN:PDF

Comisión Europea (2011b). Estrategia renovada de la UE para 2011-2014 sobre la responsabilidad social de las empresas. Recuperado de http://eurlex.europa.eu/legalcontent/ES/TXT/PDF/?ur $\mathrm{i}=$ CELEX:52011DC0681\&from=EN

Daley, J., Coyle, J, \& Dwyer, C., (2016). Sheffield Hallam University and Nestlé: Developing future leaders with the Chartered Manager Degree Apprenticeship - a partnership approach. Higher. Education, Skills and Work-Based Learning, 6(4), 370377. doi: https://doi.org/10.1108/HESWBL06-2016-0045

De Miguel, M. (Dir.) (2006). Modalidades de enseñanza centradas en el desarrollo de competencias. Orientaciones para promover el cambio metodológico en el EEES. Madrid: MEC Universidad de Oviedo. Disponible en: http://www.ulpgc.es/hege/almacen/downloa d/42/42376/modalidades_ensenanza_compet encias_mario_miguel2_documento.pdf

DOUE (2003). Recomendación 2003/361/CE de la Comisión de 6 de mayo de 2003 sobre la definición de microempresas, pequeñas y medianas empresas (DO L 124 de 20.05.3003). Recuperado de https://www.boe.es/buscar/doc.php?id=DO UE-L-2003-80730.

Egido, I. (2017). El prácticum como elemento clave en la iniciación al desarrollo profesional docente: debate y líneas de 
futuro. En H. Monarca \& B. Thoillez (coords.), La profesionalización dicente: debates y propuestas (pp. 133-147). Madrid: Síntesis.

Elijido-Ten, E. \& Kloot, L. (2015). Experiential learning in accounting workintegrated learning: a three-way partnership. Education + Training. 57(2), 204-218. doi: https://doi.org/10.1108/ET-10-2013-0122

Felce, A. (2017). The Hub in a Pub: University of Wolverhampton Apprenticeship Hub. Higher Education, Skills and Work-Based Learning. $7(1)$, 70-78. doi: https://doi.org/10.1108/HESWBL-05-2016$\underline{0035}$

Felce, A.; Perks, S. \& Roberts, D (2016). Work-based skills development: a contextengaged approach. Higher Education, Skills and Work-Based Learning, 6(3), 261-276. doi: https://doi.org/10.1108/HESWBL-122015-0058

Fernández, L. (2006). ¿Cómo analizar datos cualitativos? Butlletí La Recerca, Ficha 7, 113.

Ferrández-Berrueco, R., Kekäle, T., \& Sánchez-Tarazaga, L. (2016a). Universidad y empresa. Experiencias europeas de currículum integrado. Interrogantes pendientes. Revista Española de Educación Comparada, 27, 151-171. doi: https://doi.org/10.5944/reec.27.2016.15973

Ferrández-Berrueco, R.; Kekale, T., \& Devins, D. (2016b). A framework for work-based learning: basic pillars and the interactions between them. Higher Educatión, skills and work-based Learning, 6(1), 35-54 https://doi.org/10.1108/HESWBL-06-2014$\underline{0026}$

García Delgado, J. (2002). Lo que hemos aprendido en 20 años de prácticas en empresas. Revista de Docencia Universitaria, 2(1). Recuperado de http://revistas.um.es/redu/article/view/11841 /11421

Garnett, J. (2016). Work-based learning: A critical challenge to the subject discipline structures and practices of higher education. Higher Education, Skills and Work-Based Learning, 6(3) 305-314. doi:
https://doi.org/10.1108/HESWBL-04-20160023

Geller, J., Zuckerman, N. \& Seidel, A. (2016). Service-Learning as a Catalyst for Community Development: How Do Community Partners Benefit From ServiceLearning? Education and Urban Society, 48(2), 151-175. doi: https://doi.org/10.1177/0013124513514773

Guinart, M. (2005). Diseño de contenidos atendiendo a la diversidad formativa. La relación universidad-empresa. Estudio de caso. Revista de Universidad y Sociedad del Conocimiento, 2(2). 45-53.

Healy, A., Perkmann, M., Goddard, J. \& Kempton, L. (2014). Measuring the impact of University Business cooperation. Case studies. Luxembourg: Publications Office of the European Union.

Hegarty, P.M; Kelly, H.A. \& Walsh, A. (2011). Reflection in a workplace qualification: challenges and benefits, Journal of Workplace Learning, 23(8), 531540. doi: https://doi.org/10.1108/13665621111174889

Hernández Carrera, R.M. (2014). La investigación cualitativa a través de entrevistas: Su análisis mediante la teoría fundamentada. Cuestiones pedagógicas, 23, 187-210.

Ions, K. \& Minton, A. (2012). Can work-based learning programmes help companies to become learning organisations? Higher Education, Skills and Work-Based Learning, 2(1), 22-32. doi: https://doi.org/10.1108/20423891211197712

Kalton, G. (1983). Quantitative Applications in the Social Sciences: Introduction to survey sampling. Thousand Oaks, CA: SAGE. doi: https://doi.org/10.4135/9781412984683

Kenessey, Z. (1987). The primary, secondary, tertiary and quaternary sectors of the economy. The review of income and wealth, 33(4). 359-385. doi: https://doi.org/10.1111/j.14754991.1987.tb00680.x

Korthagen, F.A.J., Loughran, J. \& Russell, T. (2006). Developing fundamental principles for teacher education programs and practices. Teaching and Teacher Education, 22(8), 
1020-1041.

doi:

https://doi.org/10.1016/j.tate.2006.04.022

Koski, O. (2017). What work life and society do expect from applied research and innovation activity? Presentación en la Conferencia Anual de la Asociación de Rectores de universidades de Ciencias Aplicadas de Finlandia. Helsinki, 21 de noviembre.

Latorre, M.J. \& Blanco, F.K. (2011). El prácticum como espacio de aprendizaje profesional para docentes en formación. Revista de docencia universitaria, 9(2), 3554. doi: https://doi.org/10.4995/redu.2011.6157

Major, D., Meakin, D. \& Perrin, D. (2011). Building the capacity of higher education to deliver programmes of work-based learning. Higher Education, Skills and Work-Based Learning, 1(2). 118-127. doi: https://doi.org/10.1108/20423891111128890

Marcelo, C. \& Estebaranz, A. (1998). Modelos de colaboración entre la universidad y las escuelas en la formación del profesorado, Revista de Educación, 317, 97-120.

Marzo, M., Pedraja, M. \& Rivera, P. (2008). Un modelo de relaciones empresauniversidad. Revista Europea de Dirección y Economía de la Empresa. 17(1), 39-56. doi: https://doi.org/10.1016/j.redee.2013.09.003

Miles, M.B. \& Huberman, A.M. (1994). Qualitative data analysis: An expanded sourcebook. Thousand Oaks, CA: Sage.

Moliner, O. \& Sánchez-Tarazaga, L. (2015). PBL o aprendizaje basado en proyectos: una experiencia de coordinación y enseñanza auténtica en la universidad, Quaderns Digitals, 81, 116-133.

Monereo, C. (coord.) (2009). Pisa como excusa. Repensar la evaluación para cambiar la enseñanza. Barcelona: Graó.

OCDE (2012). Fostering Quality Teaching in Higher Education: Policies \& Practices. OCDE Publishing. Recuperado de http://www.oecd.org/education/imhe/QT\%2 0policies\%20and\%20practices.pdf
Senge, P. (1992). La quinta disciplina. El arte y la práctica de la organización abierta al aprendizaje. Barcelona: Granica.

Siebert, S., \& Costley, C. (2013). Conflicting Values in Reflection on Professional Practice. Higher Education, Skills and WorkBased Learning 3(3). 156-167. doi: https://doi.org/10.1108/HESWBL-07-2011$\underline{0032}$

Valle, J. \& Manso, J. (2011). Modelo para seleccionar buenos centros donde realizar las prácticas del nuevo máster de secundaria. Revista de Educación, 354, 267-290.

White, T. (2012). Employer responsive provision: workforce development through work-based learning. Higher Education, Skills and Work-Based Learning 2(2). 6-21. https://doi.org/10.1108/20423891211197703

Wengraf, T. (2012). Qualitative Research Interviewing. London: SAGE

Whittington, B. \& Ferrández-Berrueco, R. (2007). From Interaction to Integration: Developing the Relationship between Higher Education and the Labour Market. En B. Baugmartl, F. Mizikaci, \& D. Owen (eds.), From Here to There: Mileposts of European Higher Education (pp.179-196). Viena: Navreme (AT) \& Makedonska Riznitsa (MK).

Zabalza, M. A. (2008). El trabajo por competencias en la enseñaza universitaria, en VV.AA.: El nuevo perfil del profesor universitarios en el EEES: claves para la renovación metodológica, Universidad Europea Miguel Hernández, Valladolid, 79113.

Zabalza, M. A. (2011). El practicum en la formación universitaria. Estado de la cuestión. Revista de Educación, 354(1), (2143). doi: https://10.4438/1988-592X-00348082-RE

Zabalza, M. A. (2013). El practicum y las prácticas en empresas en la formación universitaria. Madrid: Narcea. 


\begin{tabular}{|c|c|}
\hline Authors / Autores & $\begin{array}{l}\text { To know more } \\
\text { / Saber más }\end{array}$ \\
\hline \multirow{5}{*}{$\begin{array}{l}\text { Ferrández-Berrueco, Reina ferrande@uji.es } \\
\text { Licenciada y Doctora en Filosofía y Ciencias de la Educación (sección CC. de la } \\
\text { Educación) por la Universidad de Valencia. Profesora Titular del Departamento de } \\
\text { Pedagogía y Didáctica de las CC. Sociales, la Lengua y la Literatura de la } \\
\text { Universitat Jaume I (Castellón, España) en el Área de Métodos de Investigación y } \\
\text { Diagnóstico en Educación. Miembro del grupo de investigación MEICRI (mejora } \\
\text { educativa y ciudadanía crítica), Centra su línea de investigación en evaluación para } \\
\text { la mejora en Educación Superior. Su dirección postal es Facultad de Ciencias } \\
\text { Humanas y Sociales. Av. de Vicent Sos Baynat s/n, 12071-Castellón de la Plana } \\
\text { (España). }\end{array}$} & \\
\hline & 0000-0001-6416-5902 \\
\hline & \\
\hline & ResearchGate \\
\hline & 0 Dial \\
\hline \multirow{4}{*}{$\begin{array}{l}\text { Sánchez-Tarazaga, Lucía lvicente@uji.es } \\
\text { Doctora en Educación por la Universitat Jaume I. Profesora Asociada del Doctora } \\
\text { en Educación por la Universitat Jaume I. Profesora Asociada del departamento de } \\
\text { Pedagogía y Didáctica de las Ciencias Sociales la Lengua y la Literatura. Centra su } \\
\text { ámbito de investigacion en la formación inicial del profesorado y el desarrollo de } \\
\text { competencias profesionales. Su dirección postal: Facultad de Ciencias Humanas y } \\
\text { Sociales. Av. de Vicent Sos Baynat s/n, 12071-Castellón de la Plana (España). }\end{array}$} & \\
\hline & \\
\hline & 0 Dialnet \\
\hline & \\
\hline
\end{tabular}

\section{RELIEVE}

Revista ELectrónica de Investigación y EValuación Educativa E-Journal of Educational Research, Assessment and Evaluation

[ISSN: 1134-4032]

Esta obra tiene licencia de Creative Commons Reconocimiento-NoComercial 4.0 Internacional.
This work is under a Creative Commons Attribution 4.0 International license. 